vernus All. begleiteten uns weiter, letzterer sogar bis auf die Spitze der Kugel.

Ein uralter Weisstannenwald mit prächtigen Stämmen nahm uns auf. Nachdem wir denselben durchwandert, betraten wir die Alpenregion der Kugel. Carex dioica und C. glauca Scop. nebst Plantago montana Lam. und Plant. alpina waren von nun an die einzigen blühenden Pflanzen, die uns bis zum Gipfelpunkto noch aufstiessen.

Der nordwestliche Vorgipfel, etwa 30 Meter niedriger, war schon ganz grün und mit Tausenden ron weissen Crocus-Blüthen übersäet.

In dem lehmigen Grunde der Kugelkuppe gedeihen Rhododendron hirsutum und ferrugineum unmittelbar nebeneinander und zugleich mit Juniperus nana Willd. und Calluna vulgaris Salisb.

Nachdem wir uns einige Stunden an der Aussicht, die vom Fluchthorn bis weit über den Bodensee hinaus reicht, und an einigen anderen nicht unwesentlichen Dingen gelabt, stiegen wir nordwärts gegen Ebrit ab.

Auf dem Wege dorthin finde ich nur zu verzeichnen Alnus viridis Gaertn., Gentiana aestiva R. S., Potentilla aurea und die hier seltene Orchis pallens. Von Ebrit stiegen wir zwischen Bocksberg und Schönenmann zur Alpe Schutana empor und trafen auf diesem Wege Primula Auricula und Veronica alpina. Auch eine sehr nette Form ron Viola arenaria DC. stand in voller Entwickelung.

Von einer auf Schutana blühenden Gagea habe ich in der Eile leider so unvollkommene Exemplare mitgenommen, dass ich mit Sicherheit nicht sagen kann, ob es $G$. Liottardi Schult. oder $G$. pusilla Schult. ist. Von Schutana bis auf die Alpe Kühberg traf ich noch Viola biflora $\mathrm{L}$. Von dort ab geht es durch düstere Tannenwälder, die für den Botaniker wenig Bemerkenswerthes bieten, abwärts nach Dornbirn.

Feldkirch, am 12. Mai 1880.

\title{
Die Veilchen des Bisamberges bei Wien am 6. April 1880.
}

\section{Von Josef Wiesbaur S. J.}

Die von Herrn Heimerl voriges Jahr am Bisamberge entdeckte Viola ambigua $W K$. veranlasste mich, diesen in Wiens Nähe gelegenen interessanten Berg einmal zu besuchen. Wo Viola ambigua wächst, dachte ich mir, mag es auch andere Veilchen geben. Ich hatte mich hiebei, wie folgende Zeilen zeigen werden, nicht verrechnet. Bald nach 12 Uhr (am 6. April) konnte ich in Langenzers- 
dorf die Bahn verlassen und hatte num bis 4 Uhr Zeit, auf den nächsten Anhöhen über dem Dorfe zu suchen. Zwar gelang es mir nieht, das sehönste unserer wohlriechenden Veilchen, die Viola Haynaldi aufzufinden, obschon ich es darauf zunächst abgesehen hatte. Uebrigens wird diese schöne Pflanze böchst wahrscheinlich noch hier entdeckt worden. Ich wurde, obgleich der fast beständige Regen meine Absichten wenig begünstigte, anderweitig ontschädigt.

Zunächst traf ich am westlichen Ende des Dorfes die Viola austriaca Kerner. Sie scheint hier auf der Südseite des Berges in den Gebüschen über und in den Weingärten allein vorzukommen ${ }^{1}$ ) und auch am linken Donauufer über Berg und Ebene verbreitet zu sein, wie sie es am rechten ist. Vor drei Jahren verfolgte ich sie von Laxenburg über Münchendorf, Moosbrunn, Grammatneusiedl und Reissenberg bis ins Leithagebirge, wo ich sie bei Mannersdorf, Somerein und Kaisersteinbruch (Ungarn) traf und am Rückweg wieder bei Wilfleinsdorf, Himberg, Lanzendorf, Leopoldsdorf, Hennersdorf, Vösendorf und Siebenhirten bis Liesing beobachtete.

Viola hirta ist natürlich auch über den ganzen Bisamberg verbreitet. Bald traf ich am kleinen Fussweg, der mich den Berg hinanführte, ein ziemlich stark rasenbildendes sonst der Viola austriaca sehr ähnliches Veilchen, welches mir schon aus Kalksburg, Mödling und Laxenburg bekannt war, und das ich für $\boldsymbol{V}$. austriaca $\times$ hirta halte. Das Vorkommen am Bisamberge, wo es unter den beiden genannten Arten allein wächst, bestätigt meine frühere Annahme. Ich erlaube mir dafür die Benennung Viola Kerneri in Vorschlag zu bringen, so dass es den Namen desjenigen trage, durch dessen verdienstvolle Studien wir zuerst unsere Viola austriaca von der $\boldsymbol{V}$. suavis des Kaukasus unterscheiden lemten. Viola Kerneri scheint sehr verbreitet zu sein, wahrscheinlich ist sie überall dort, wo Viola austriaca und $\boldsymbol{V}$. hirta zugleich vorkommen. Vom Leithagebirge (Kaisersteinbruch) und vom Johannesberg bei Ofen cultivire ich sie. Es scheint zwei Formen derselben zu geben. Die eine (superaustriaca $\times$ hirta), welche ich am Bisamberge fand, ist leichter zu erkennen. Sie hat ganz die Blumenfarbe der Viola austriaca, ist anch im Schlunde weiss wie diese, jedoch fast geruchlos. Die andere Form (superhirta $\times$ austriaca) ist sehwer von der Viola permixta Jord. (hirta $>$ odorata), namentlich von deren Form superhirta $>$ odorata zu unterscheiden. Sie hat zWar wie die erste Form tiefgestellte Deckblättchen (Hochblätter), schmale Nebenblätter, die fast so lang gefranst sind, wie die an $V$. austriaca, aber auch an $V$. permixta, namentlich an der erwähnten Form superhirta >odorata kann man das öfters treffen, so dass es an Standorten, wo Viola odorata, austriace und hirtla zugleich vorkommen, kaum gelingen dürfite, durch rein morphologische Merkmale die beiden genannten Formen zu unterscheiden.

1) Die Viola odorata traf ich nur am Bächlein am östlichen Ende des Dorfes und in den Auen. 
Noch beror ich den halben Berg erstiegen hatte, gewahrte ich jenseits eines dornenreichen Grabens ein viertes Veilchen. Dem musste ich beikommen. Es war Viola hybrida Val de Lièvre (Oe. b. Z. 1858, S. 59) $=V$. collina $>$ hirta und gleich daneben abwechselnd mit $V$. hirta die nun sicher erwartete $V$. collina selbst in ziemlicher Menge. Auffallenderweise ist diese in Neilreich's Flora von Nied.Oesterr. vom Bisamberg nicht angegeben. Der verdienstvolle Autor dieser Flora kann demnach zur Zeit, wo $V$. collina blüht, niemals hier gewesen sein. Er würde diese Pflanze, welche ihm sehr gut bekannt war, nicht ïbersehen haben.

Der Regen hatte etwas nachgelassen, und ich begann nach der mir aus der Kalksburger Gegend bekannten $V$. collina $\times$ arsstriaca (V. suaveolens $\mathrm{m}$. ined.) zu suchen; jedoch vergebens, vielleicht desshalb vergebens, weil $V$. austriaca in der oberen Region des Bisamberges zu selten ist. Suchend gelangte ich num an die Grenze der Brandfläche; am Charsamstag war nämlich, wie mir Arbeiter erzählten, die ganze obere südwestliche Berggegend durch Leichtsinn oder Muthwille in Brand gerathen. Auf Viola ambigua hatte ich bereits, mit den erwäbnten fünf Veilchen zufrieden, zu verzichten begdnnen. Da bemerkte ich hart an der Brandgrenze ein grossblumiges tief rothviolettes Veilchen prangen; es war die stark duftende Viola ambigua W. et $\mathrm{K}$., welche im letzten Jahre auch in Ungarn von Herrn Dr. J. A. Tauscher bei Ercsi an der Donau entdeckt ward, wie jch mich an gütigst übersandten Exemplaren zu überzeugen Gelegenheit hatte. Die hiesigen Pflanzen stimmen ganz mit den ungarischen. Am Bisamberg ist das Zusammentreffen der Viola ambiaua und $V$. collina von grossem Interesse, und verdient in günstigen Jahren weiter verfolgt zu werden. $O b$ es wohl auch, wie bei den übrigen Arten, eine unfruchtbare Mittelform, eine $V$. ambigua $\times$ collina geben mag? Heuer hat der Wiesenbrand zu sehr geschadet, um mit Erfolg weiter suchen zu können, anch hatte es mittlerweile wieder zu regnen begonnen; desshalb wandte ich mich, Wind und Regen den Rücken kehrend, nach Osten, wo ich in der oberen Region Viola ambigua mit $V$. hirta häufiger anftroten sah. Auch die schon am Mödlinger Eichkogl beobachtete $V$. ambigua $\times$ hirta, die ich als $V$. hirtaeformis zu bezeichnen pflege, fehlte hier nicht. Blühend hat sie eher das Aussehen einer $V$. umbigua, als das einer $\nabla$. hirta, ist aber fast ganz geruchlos. In Blättern und Nebenblättern nähert sie sich mehr der $\hat{V}$. hirta und wächst unter den Stammarten auf freier Wiesenfiäche. Ausser den erwähnten sieben Veilchen der Gruppe Viola Martii war in der oberen Region anch die grössere Form der $V$. arenaria, die $V$.rupestris Schm. bereits in Blüthe. Ich eilte nun bergab zum Bächlein unter den Steinbrüchen. Bis nun war das Unterscheiden der verschiedenen Veilchen nicht schwer gewesen. Am Bächlein traf ich auf $V$. odorata L., und mit dem Auftreten dieser Pflanze zugleich mit $V$. austriace und $V$. hirta begannen die Schwierigkeiten, denen ich überall zu begegnen pflege, wo diese drei Arten, und sind es auch nur sio allein, bei- 
sammen stehen. Viola permixta Jord. traf ich hier am Bächlein heute zum ersten Male, worin ich einen neuen Beweis erblieke, dass sie der Verbindung $V$. hirta $\times$ odorata entspreche. Ob aber alles, was hier $V$. permixta zu sein schien, es auch war, ist schwer zu entscheiden, da höchst wahrscheinlich auch die schon oben erwähnte zweite Form der $V$. Kerneri, dio $V$. superhirta $\times$ austriaca, sich darunter befand. Es gibt wohl auch eine Form der V. permixta, die leicht $z u$ erkemnen ist. Es ist das die der $V$. odorata näher stehende Pflanze, der sie blühend fast ganz gleich ist, namentlich was die Blumenfarbe betrifft. Sio unterscheidet sich aber durch geruchlose oder nur kurze Zeit schwach wohlriechende Blumen, spitzeres Lanb und sehr kurze Läufer. Ausser dieser Viola subhizta $\times$ odorata gibt es auch eine $V$. superhirta $\times$ odorata, von der bereits oben die Rede war, und welche von der blasseren Form der Viola Kerneri, von der $V$. superhirta $>$ austriaca sich so schwer unterscheiden lässt.

Eine weitere Schwierigkeit bietet die Verbindung von Viala austriaca $\times$ odorata. Ich fand diese Mischart zuerst in der Brigittenau, dann auf der Schmelz und 1879 am Himmel bei Wien und schlage ich desshalb dafür die Benennung Viola vindobonensis vor. Es scheint dieses Veilchen gleichfalls überall vorzukommen, wo die Stammarten sich finden. Es ist aber jedenfalls dort leichter zu erkennen, wo andere wohlriechende Veilchenarten (wie Viola alba, $V$. cullina) fehlen, z. B. in Laxenburg, wo $V$. ausiriaca und $V$. odorata sehr häufig sind. Die tiefer gestellten Deckblätter, die stärker gefransten Nebenblätter unterscheiden sie von $V$. odorata. Die Farbe der Krone hingegen nähert sich dem Rothviolett der $V$. odorata, hat aber noch ziemlich viel vom weissen Schlund der blauvioletten V. austriaca (Sturm's Bild der V. Steveni in Deutschlands Flora seheint mir, was die Blumenfarbe betrifft, ziemlich gut der $V$. vindobonensis zu entsprechen). Neben solchen echten Mittelformen scheinen aber auch andere vorzukommen, welche bald der $V$. austriaca, bald der $V$. odorata sich nähern und die sehr schwer zu deuten sind. Auch die Cultur bietet auf dem sehr veilchenreichen Boden von Kalksburg selbstverständlich grosse Schwierigkeiten dar. Das Wetter war nicht darnach, den Schwierigkeiten auf den Grund zu gehen. Ich zog vor unter Dach za kommen. Jedoch eine ganze Stunde im Bahnhof za warten (es war erst $3 \mathrm{Ubr}$ ) war zu langweilig. Ich ging daher in die Au hinaus und fand nächst dem Stationsgebäude folgende Veilchen: Viola hirta, odorata, austriaca, Kerrieri, pesmixta und zu meiner Ueberraschung auch $V$. collind. Ich hatte diese Art bisher nur auf Bergabhängen gefunden und sie in einer DonauAu am wenigsten erwartet.

Das Resultat des Bisamberg-Besuches war somit ein höchst überraschendes: 10 verschiedene Veilchen. Sonst war wenig Anlockendes zu sehen. Alles, was ich auf meinem Woge bemerkte, waren viele Carex humilis, viel Pulsatilla patula Pritzel var. (Anemone pratensis Noilr.) und Fulsatilla grandis Wenderoth (An. 
Pulsatilla Neilr.) und zwar, wie auch überall um Kalksburg, in bei den Formen, was die Färbung der Haare betrifft, f. chrysothrix und f. argyrothrix. Ferner je ein Exemplar von Iris pumila (gelbe Form) und von Primula officinalis f. Pr. inflata Lehm. (ein gynodynamisches Exemplar) nebst viel Potentilla cinerea Neilreich. Am Bächlein gab es Pulmonaria officinalis L. (unter mehreren gefleckten auch ein ungeflecktes Exemplar). In der Au ausser $G a$ lanthus und Gagea silvestris anch bereits Oxalis Acetosella, dio nach $\mathrm{N}$ eilreich in den Donau-Auen nur zerstreut anftritt.

Für Veilchenstudien scheint aber der Bisamberg sehr geeignet zu sein, da fast nirgends viele Arten zugleich anftreten. Auch fehlen auf dern von mir eingeschlagenen Wege wenigstens - Viola alba Bess. (und V. scotophylla Jord., welche, wie Prof. G. Strobl in dieser Zeitschrift 1877, S. 222 richtig bemerkt, mit Recht zur $V$. alha gezogen werden muss, und deren Formen mir trotz Jor dan nur Farbenspielarten der $V$. alba Bess. sind) sowie die damit zusammenbängenden $V$. multicaulis, $V$. badensis und $V$. kalksburgensis gänzlich. Es ist dadurch die grosse Verwirrung nicht za fürchten, welche im Kahlengebirge, im Leithagebirge, auf den Hainburger und Presburger Bergen u. s. w. durch $V$. alba und ihr Gefolge angerichtet wird, welcher Umstand auch grossentheils Schuld trägt, dass diesen sonst so beliebten Gewächsen von Fachmännern nicht die gebührende Anfmerksamkeit geschenkt worden ist.

Kalksburg, am 10. April 1880.

\section{Veber}

\section{die Einbürgerung exotischer Unkräuter und anderer Pflanzen in Süd-Australien von Dr. Rich. Schomburgk.}

\section{Tebersetzt von F. Antoine.}

(Behiuss.)

Von den Leguminosen haben sich folgende jedoch zur Verbesserung des Wiesenlandes verbreitet: Trifolium repens L., $T$. agrarium L., T. pratense L., Melilotus parviflorus Desf., Medicago sativa Dec., Med. denticulata Willd., Vicia sativa L., V. hirsuta Fisch.

Foeniculum vulgare Gaertn. Diese nützliche Medicinalpflanze ist schon vor langer Zeit aus Europa hierher gebracht worden und hat sich auf eine erstaunliche Weise im Lande verbreitet, besonders liebt sie als Standort die Ufer der kleinen Flüsse, wo sie oft zur enormen Höhe von 4 bis 6 Fuss emporwächst, förmliche Dickichte formirt und die krautartigen Pflanzen unterdrüekt. 